\section{Die Allergie raucht immer mit}

\section{Tabakrauch gilt als gesicherter Kofaktor bei der Genese atopischer Erkrankungen. Allerdings gibt es kaum Studien, bei denen der direkte Zusammenhang zwischen Rauchinhalation und der Reaktion auf eine unmittelbar folgende Allergenexposition untersucht worden ist.}

K alifornische Forscher ließen 19 junge, gegen Ragweed sensibilisierte Nichtraucher in einer speziellen Expositionskammer unter kontrollierten Bedingungen entweder Zigarettenrauch oder rauchfreie Luft einatmen. Danach erfolgte eine nasale Provokation mit Ragweed-Pollen. Vor sowie in verschiedenen Abständen von bis zu sieben Tagen nach der Exposition wurden Nasensekretproben durch eine Lavage entnommen.

Wie erwartet, war nach Pollenexposition das spezifische $\operatorname{IgE}$ im Nasalsekret signifikant angestiegen. Bei Teilnehmern, die zuvor zusätzlich zwei Stunden
Rauch inhaliert hatten, war dieser Anstieg allerdings ungleich höher. Das spe-

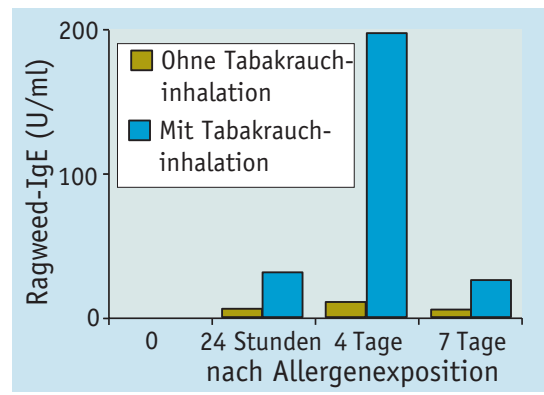

Spezifisches IgE in der Nasallavage nach Ragweed-Exposition

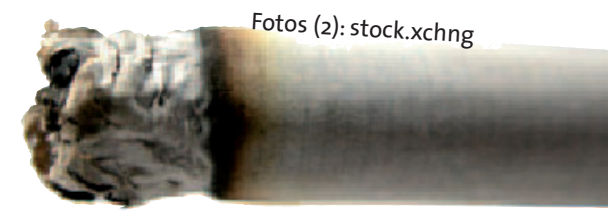

zifische $\mathrm{IgG}_{4}$ war in der Gruppe mit Tabakrauchinhalation ebenfalls signifikant höher. Ähnliches galt für die Zytokine IL-4, -5 und -13: Sie waren nach Ragweed-Exposition ohne Rauch geringfügig, mit Rauch signifikant häufiger in der Nasallavage zu finden. Interferon- $\gamma \mathrm{da}-$ gegen fiel unter zusätzlicher Rauchexposition deutlich ab. Histamin schließlich nahm nach Ragweed-Provokation ohne Rauch um den Faktor 7,7, mit Rauch um den Faktor 25,1 zu.

Fazit: Diese experimentelle Studie belegt deutlich, dass Tabakrauch in der Umgebungsluft die allergische Reaktion bei entsprechend sensibilisierten Patienten verstärken kann.

Diaz-Sanchez D et al. Challenge with environmental tobacco smoke exacerbates allergic airway disease in human beings. J Allergy Clin Immunol 2006; 118: 441-6

\title{
Ausweg aus dem Sucht-Teufelskreis?
}

\section{Die Raucherentwöhnung mit Nikotinersatzpräparaten oder dem Anti- depressivum Bupropion zeigt meist nur mäßigen Erfolg. Als effektiv hat sich jetzt in drei Studien die neue Substanz Vareniclin erwiesen.}

V areniclin ist ein partieller Agonist des $\alpha 4 \beta 2$-nikotinergen Azetylcholinrezeptor(nAChR)-Subtyps und besitzt damit ein duales Wirkspektrum: Durch die partielle Aktivierung des $\alpha 4 \beta 2$-nAChR werden zum einen die Entzugssymptome durch Freisetzung von Dopamin gemildert, zum anderen blockiert Vareniclin als partieller Antagonist einen Teil des Nikotinrezeptors und damit die Bindung von inhaliertem Nikotin. Dadurch wird der Teufelskreis durchbrochen, der über das Wohlbefinden während einer aktuell gerauchten Zigarette schon das Verlangen nach der nächsten Dosis programmiert.

Dass diese pharmakologischen Effekte klinische Konsequenzen haben, 1) zeigen zwei methodisch ähnliche, randomisierte und plazebokontrollierte Ver- gleichsstudien [1, 2] mit Vareniclin versus retardiertem Bupropion. Beteiligt waren 1.025 bzw. 1.413 Raucher, die 12 Wochen lang die Medikamente erhielten und dann über 52 Wochen weiter beobachtet wurden. In beiden Studien war Vareniclin Plazebo und Bupropion sowohl während der aktiven Intervention als auch in der Nachbeobachtungszeit signifikant überlegen. Häufigste Nebenwirkung unter Vareniclin war Übelkeit, einige Patienten brachen die Behandlung deshalb ab.

In einer dritten Studie [3] wurde zusätzlich der präventive Effekt von Vareniclin bei entwöhnten Rauchern gegenüber einer Wiederaufnahme der Sucht untersucht. Dazu wurden 1.927 Raucher zunächst offen 12 Wochen lang mit Vareniclin behandelt. Die 1.236 Probanden, die danach nicht mehr rauchten, wurden randomisiert einer weiteren zwölfwöchigen Behandlung mit Vareniclin oder Plazebo zugewiesen und insgesamt 52 Wochen beobachtet. Ergebnis: Die präventive Wirkung von Vareniclin war der von Plazebo während der Nachbeobachtungszeit signifikant überlegen.

Fazit: Der partielle $\alpha 4 \beta 2$-nAChR-Agonist Vareniclin ist in der medikamentösen Raucherentwöhnung Bupropion überlegen. Bei bereits entwöhnten Rauchern konnte ein präventiver Effekt gegen einen Rückfall gezeigt werden. $\quad b k$

1. Gonzales D et al. Varenicline, an $\alpha 4 \beta 2$ nicotinic acetylcholine receptor partial agonist, vs sustained-release bupropion and placebo for smoking cessation: a randomized controlled trial. JAMA 2006; 296: 47-55

2. Jorenby DE et al. Efficacy of varenicline, an $\alpha 4 \beta 2$ nicotinic acetylcholine receptor partial agonist, vs placebo or sustainedrelease bupropion for smoking cessation: a randomized controlled trial. JAMA 2006; 296: 56-63

3. Tonstad $\mathrm{S}$ et al. Effect of maintenance therapy with varenicline on smoking cessation: a randomized controlled trial. JAMA 2006; 296: 64-71 